\title{
Forbush-storm classification of the events as a device for the solar wind diagnostics
}

\author{
Lyudmila P. Shadrina ${ }^{11}$ \\ ${ }^{1}$ Academy of sciences of Sakha (Yakutia) Republic, Yakutsk, Russia
}

\begin{abstract}
The geoeffectiveness of the solar wind is usually determined on Earth by such phenomena in the geomagnetic field as storms and substorms. The second, no less significant effect is a sharp decrease in the intensity of galactic cosmic rays (GCR) Forbush effects. A joint examination of these effects in the geomagnetic field and in the GCR makes it possible to obtain additional diagnostic features, since they carry different information. The behavior of the geomagnetic field reflects changes in the magnetosphere, while the GCR intensity depends on the spatial configuration of the magnetic field in the heliosphere. To carry out such work, it is proposed to use the Forbush-Storm classification - a catalog of geophysical events in the geomagnetic field and cosmic rays. The catalog shows the dates and time of the beginning of the events of a decrease in the Dst geomagnetic field index and in GCR intensity from 1996 to 2017, for 2 cycles of solar activity. It is shown that these two types of terrestrial manifestations of interplanetary disturbances can occur simultaneously or separately.
\end{abstract}

\section{Introduction}

This paper describes one of the possible methods for diagnosing the interaction of disturbed structures of the solar wind and the Earth's magnetosphere on the basis of ground-based observations of the geomagnetic field and galactic cosmic rays (GCR). There are two ways of studying the impact of sporadic and recurrent solar wind streams to Earth. The first is the investigation of geomagnetic phenomena - storms and substorms [1-10], the second is the analysis of GCR variations, including Forbush effects - decreases in the GCR intensity during geomagnetic storms [11-15].The study of geomagnetic phenomena is based on the analysis of magnetospheric phenomena. This is a kind of analysis of a disturbed flow impact in one point - at the location of the Earth in the heliosphere. The study of GCRs carries information about all the heliosphere, and in particular on the state of interplanetary magnetic field (IMF), since GCR intensity variations on Earth are determined by these parameters. A number of geomagnetic storm catalogs and some lists of Forbush effects have been published [see for example 16-20].

It is shown in [21-23] that a joint analysis of these two ground-based manifestations of the disturbed solar wind makes it possible to consider events from two points of view.

\footnotetext{
1 *Corresponding author: $\underline{\text { lushadr@mail.ru }}$
} 
In papers [24-26], Forbush-decreases and geomagnetic storms data are used for the investigation of disturbed solar wind structures, but usually such works focus their attention on events of high intensity. The main distinction of our approach is that we take into account not only intense, but also moderate and weak events [27]. This provides additional information that makes it possible to obtain a more complete picture of the interaction of solar wind disturbed structures with the Earth.

\section{Data}

Forbush-storm classification by ground-based events was proposed and substantiated in papers $[21,22,27]$. The catalog is the result of geomagnetic storms and Forbush effects studies carried out since 1980s. It includes 22 sheets from 1996 to 2017, each containing the information on the geomagnetic storms and GCR intensity decreases. In total, the catalog includes data on 1193 storms and 1551 Forbush effects.

Geomagnetic storms are determined based on the Dst geomagnetic activity index, which is available online on the website of Data Analysis Center for Geomagnetism and Space Magnetism Graduate School of Science, Kyoto University[28]. We consider as geomagnetic storm a decrease of the Dst index by $30 \mathrm{nT}$ and more, up to $400 \mathrm{nT}$ during 4-14 hours (the main phase of the storm) and then Dst returns to its previous level within 10 hours to several days. According to the magnitude of this Dst decrease storms are divided into weak ones (30$50 \mathrm{nT}$ ), moderate (50-100 nT), strong (100-200 nT) and extrastorms (more than $200 \mathrm{nT}$ ) [5]. The catalog includes 5 parameters characterizing geomagnetic storm: the storm number, date, day of the year (to agree with King's catalog; January, 1 is 0 day; December,31 is 364 day), hour of the beginning of the storm's main phase, UT, the main phase duration $\Delta \mathrm{T}$, in hours (interval from the maximum to the minimum of Dst index) and storm amplitude $\Delta \mathrm{Dst}$, in $\mathrm{nT}$. The beginning of the storm is the hour when the Dst index reaches the maximum value before the main phase decrease. The amplitude of a geomagnetic storm is the difference between the maximum and minimum values of the Dst index: $\Delta \mathrm{Dst}=-\left(\mathrm{Dst}_{\max }-\mathrm{Dst}_{\min }\right)$.

Forbush effect is a decrease in GCR intensity recorded by the ground-based muon detector network. Forbush-decreases data are presented for example on the website of the IZMIRAN Space Weather Forecast Center [20], together with parameters characterizing the state of the interplanetary medium. They assume a Forbush effect is an event of GCR intensity decrease with $\Delta \mathrm{I} \geq 0,4 \%$. Our catalog includes events with $\Delta \mathrm{I} \geq 0,8 \%$ and greater. Three parameters are included: date, hour of the beginning of the event, UT and the value of the GCR decrease $\Delta \mathrm{I}$.

We have introduced the concept of three classes of ground events: registration of Forbushdecreases without geomagnetic storms (class 1, F - S), observation of Forbush-decreases together with geomagnetic storms (class $2, \mathrm{~F}+\mathrm{S}$ ), or geomagnetic storms without Forbushdecreases

(class 3, S - F) [21].

Fig. 1 shows an example from the catalog: a list of Forbush-Storm classification of the events in 2017. The first 6 columns (Fig. 1a) contain data on geomagnetic storms; the next 3 columns - data on GCR intensity decreases; and then three columns are counting the number of events of each class (1 means the presence an event of one of the three classes, $\mathrm{F}-\mathrm{S}, \mathrm{F}+\mathrm{S}$ or S - F). The last line of each table (Fig. 1, c) shows the results of calculating the annual average values of the main phase duration, the intensity of geomagnetic storms and GCR decreases, and the number of events of each class. These results are also presented on the histogram showing the ratio between the three classes of events (Fig. 1b). 
The last, 23rd sheet of the catalog contains a table with the summary results for 19962017, i.e. for two cycles of solar activity, 23rd and 24th (Fig. 2).

This sheet also contains a graphic image of these summary results, i.e. the number of all 3 classes of Forbush-Storm events together with the solar activity curve.
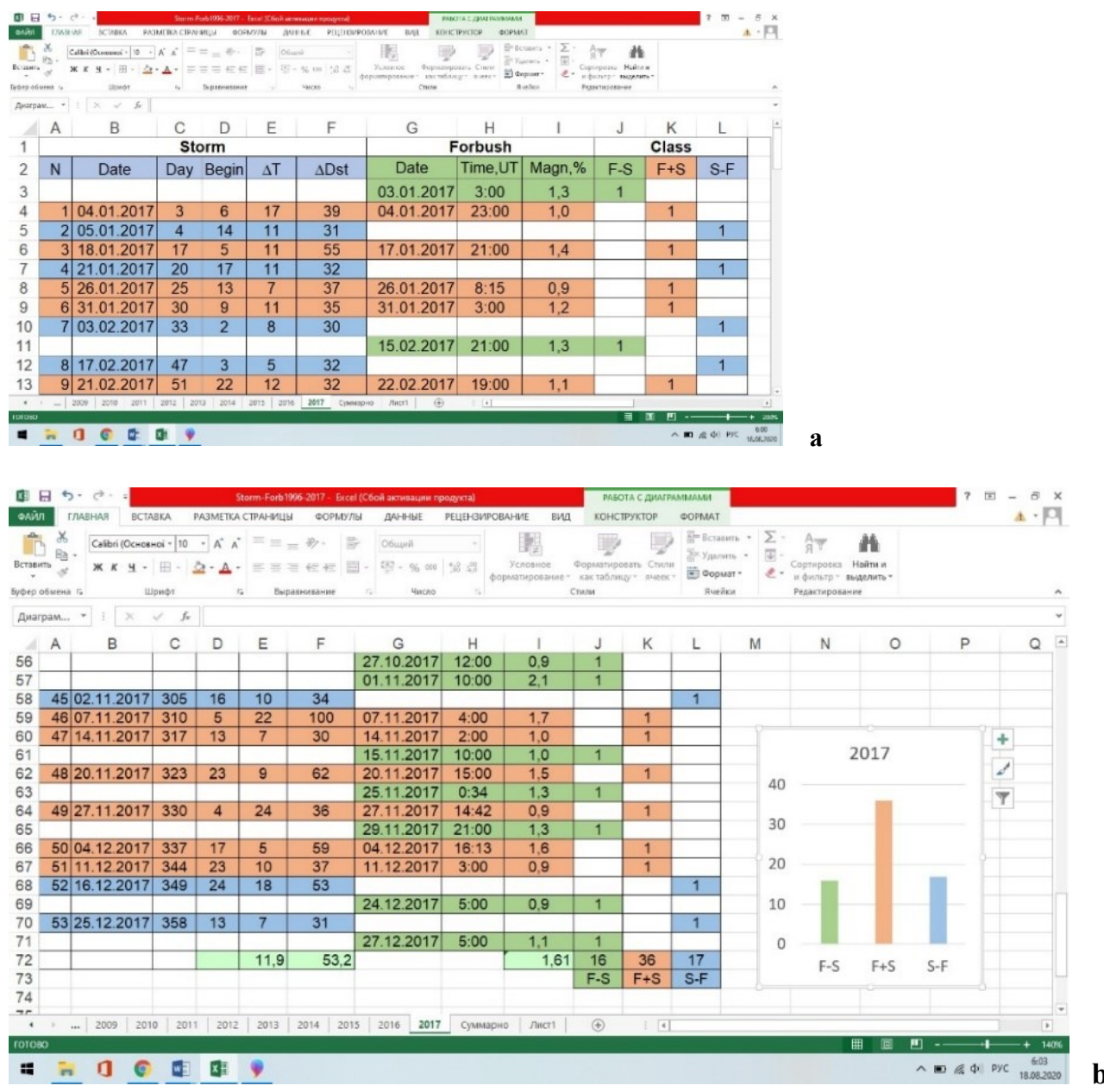

Fig. 1. An example of a sheet from the catalog of Forbush-Storm classification of events in 2017: the beginning of the table (Fig. 1a, above) and the end (Fig. 1b, below).

\section{Results and discussion}

In papers $[22,27]$ we carried out the analysis of the interplanetary parameters behavior for three classes of the Forbush-Storm classification. The result was the assumption that three classes ground-based events exist due to different trajectory of Earth through the disturbed solar wind flux.

Class 1 of events ( $\mathrm{F}-\mathrm{S}$, Forbush-decreases without geomagnetic storms) occur when Earth crosses the western flank of the disturbed solar wind flux. In this case, the Earth falls into an area with a reduced content of cosmic rays (Forbush-decrease region) and does not 
enter the storm-causing area. It is believed that the cause of the geomagnetic storm is high values of the southern IMF (Bs) in magnetic clouds associated with the interplanetary coronal mass ejections (ICMEs) or corotating interaction regions (CIRs). Due to the quasi-spiral configuration of the IMF, the Forbush-decrease region may to be displaced to the western flank of the disturbed solar wind structure.

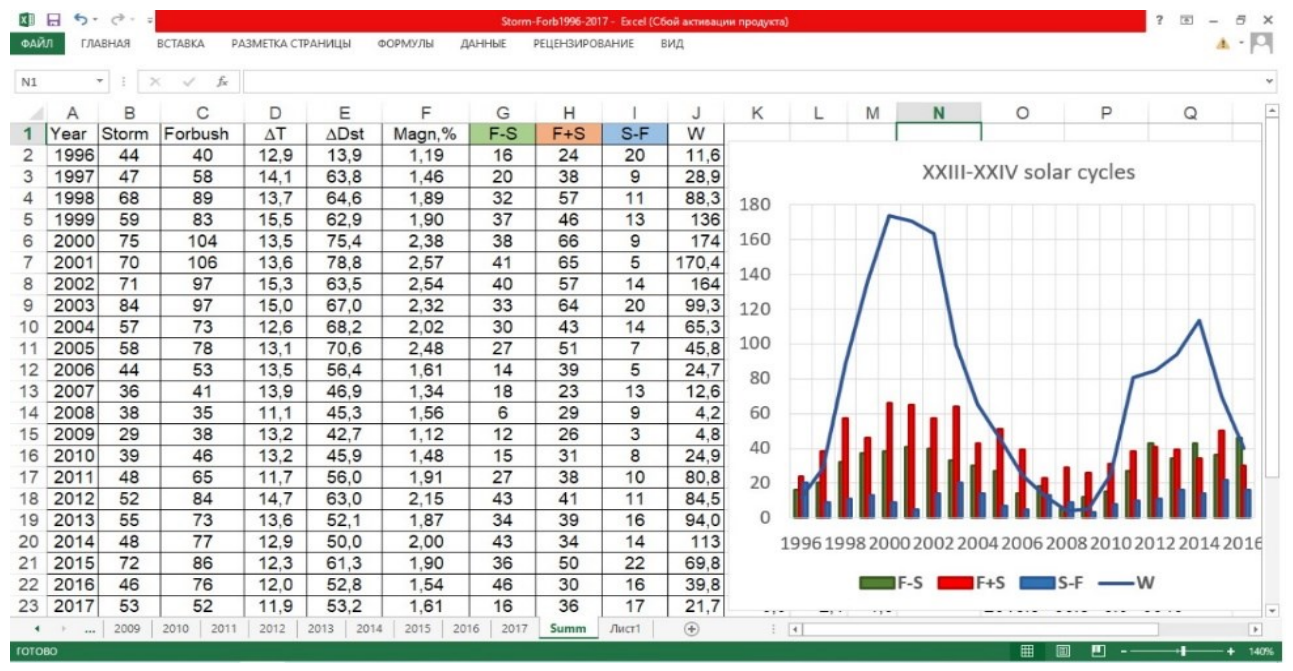

Fig. 2. A sheet of summary values of Forbush-Storm events: the number of geomagnetic storms (Storm) and Forbush decreases (Forbush) for each year, average values of storm main phase duration $\Delta \mathrm{T}$, the intensity of storms $\Delta$ Dst and Forbush decreases $\Delta \mathrm{I}$, the number of the events for each of the three classes of events (F - S, F + S, S - F) and average annual solar activity (W - Wolf number, sunspot number) for the XXIII and XXIV solar cycles.

Class 2 of events ( $\mathrm{F}+\mathrm{S}$, Forbush-decreases together with geomagnetic storms) occur at the central intersection of the solar wind flows. In this case, Earth enters Forbush-decrease region and ICME with a magnetic cloud containing the southern IMF component, which causes geomagnetic storms.

Class 3 of events ( $\mathrm{S}-\mathrm{F}$, geomagnetic storms without Forbush-decreases) are associated with eastern flank intersections. In this case, the Earth receives into the ICME with a magnetic cloud and past the Forbush-decrease region.

These conclusions partly agree with the results of papers $[13,25,29,30]$. They studied the dependence of Forbush effects on the heliolongitude of the flares causing them.

To illustrate the difference in the interplanetary medium state of the three Forbush-Storm classes, we show the behavior of the Dst index, CR intensity in Yakutsk, 2 solar wind parameters and 3 IMF parameters for the events in 1999 (Fig. 3).

One can see that for the event of $1^{\text {st }}$ class on June, 26-28 there is no geomagnetic storm. Dst index changes from -4 to $+38 \mathrm{nT}$, but there is Forbush-decrease of GCR intensity by more than $3 \%$. The solar wind velocity changes insignificantly, but its abrupt increase during the splash in the solar wind density is noticeable. This may be considered as an evidence of crossing a shock. IMF parameters - module, Bz component, and variability sigB - show small changes. But it is necessary to note an increase in IMF modulus at the time when the solar wind density increase, i.e. during crossing a shock. Such changes can be interpreted as crossing the western flank of a disturbed solar wind flow. 
For the event of $2^{\text {nd }}$ class on February 18-20, there are all signs of a central intersection of a disturbed flow: an increase in the solar wind velocity and density, a significant increase in the IMF module, large negative values of the Bz component, and growth in IMF variability sigB, which is associated with IMF turbulence. The behavior of ground-based parameters (the Dst index and the GCR intensity) are typically for a large geomagnetic storm with an amplitude of about $120 \mathrm{nT}$ and a Forbush-decrease of almost $6 \%$.
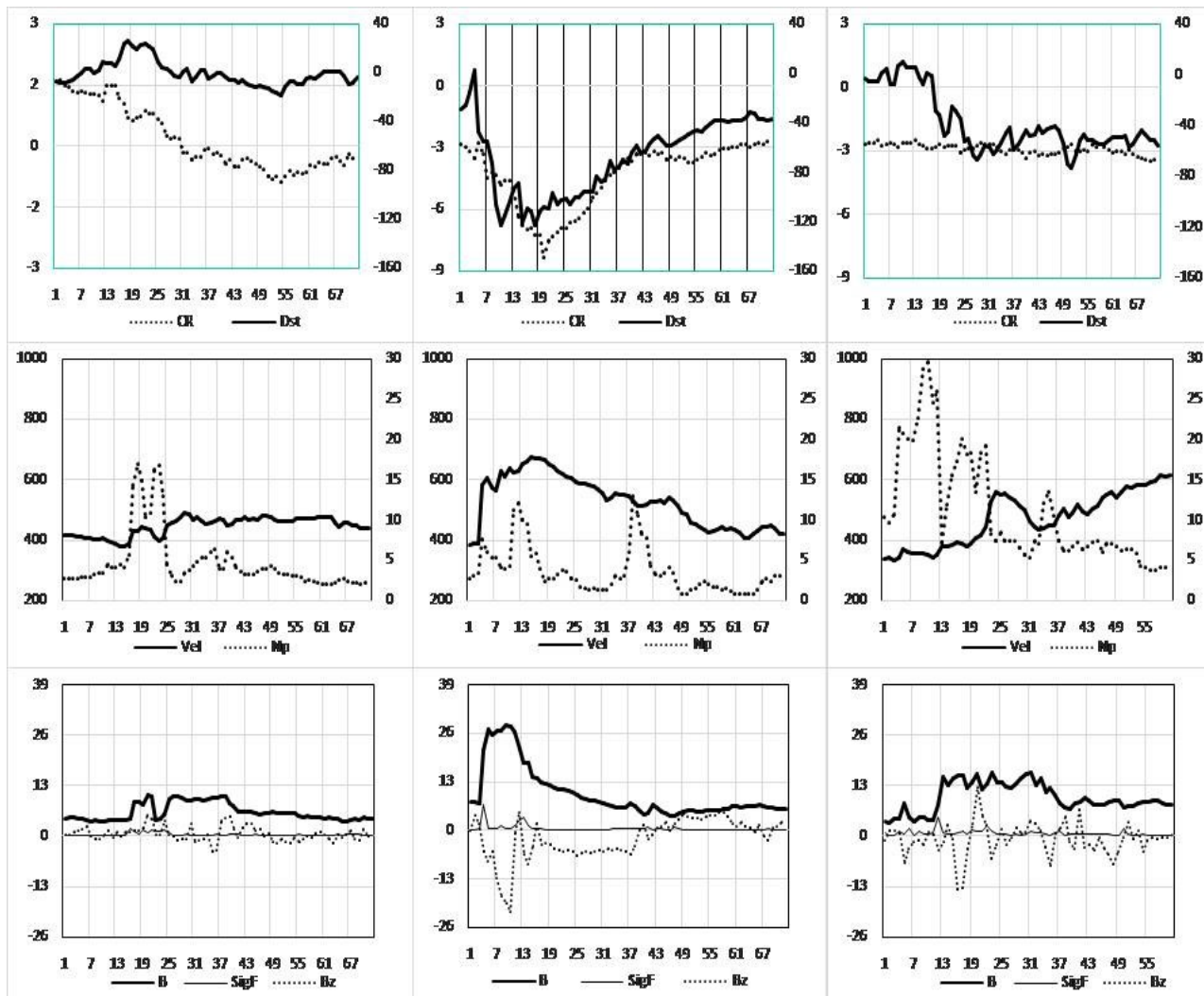

June, 26-28

February, 18-20

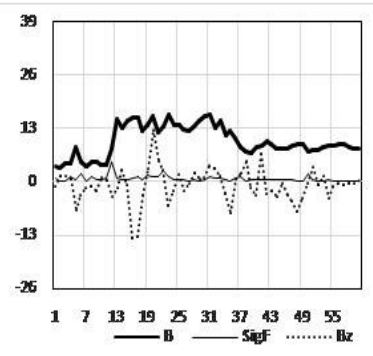

November, $6-8$

Fig. 3. An example of interplanetary parameters behavior in three classes of Forbush-Storm classification for events in 1999. Class 1, June, 26-29, CR decrease without a geomagnetic storm; Class 2, February, 18-20, Forbush decrease together with a geomagnetic storm; Class 3, November 6-8, geomagnetic storm without a CR decrease. Upper row, geomagnetic Dst index and CR intensity; middle row, velocity (Vel) and density $(\mathrm{Np})$ of the solar wind; bottom row, IMF parameters: value (B), variability (sigB) and Bz-component.

For the event of $3^{\text {rd }}$ class on November 6-8 we see a moderate geomagnetic storm with amplitude of Dst index more than $40 \mathrm{nT}$, but the GCR intensity remains at one level. There are significant changes in the solar wind parameters - several big splashes in solar wind density and a gradual increase in speed by the value of about $200 \mathrm{~km} / \mathrm{s}$. The IMF module shows a sharp increase, the Bz component changes from large negative to positive values these are signs of crossing the magnetic cloud. Growth of IMF variability sigB at the leading edge of the magnetic cloud is a sign of noticeable turbulence. 
Thus, this example shows that in the three classes of the events, the behavior of interplanetary parameters is significantly different. This allows us to consider the ForbushStorm classification of events as a device for determining how the Earth's trajectory passes through the solar wind flow. We understand the word device in a means of a method for determining the geometric factor of the Earth's intersection of the disturbed solar wind flux based on measurements of the geomagnetic field and GCR intensity.

Predicting geomagnetic storms is often complicated by the question of why intense flares do not generate strong geomagnetic storms. And the second problem is to select an active structures on the Sun - flare, or prominence, or coronal hole - as the reason of geomagnetic storm and Forbush-decrease on the Earth. In our opinion, the explanation for this is precisely the geometrical factor of the Earth's getting into ISME or CIR.

A disturbed solar wind flux can produce different effects in geomagnetic field and GCR intensity depending on how the Earth crosses this flux - at center or at flanks. If geomagnetic storm and GCR-decrease are observed together, i.e. event of the $1^{\text {st }}$ class $(\mathrm{F}+\mathrm{S})$ is observed, it can be concluded that the Earth crosses the disturbed flow at center. If a Forbush-decrease is observed without a geomagnetic storm, this is $2^{\text {nd }}$ class $(\mathrm{F}-\mathrm{S})$ event, and it can be assumed that the Earth is crossing the flow on the western flank. And, probably, its source is an active region on the eastern flank of the solar disk. Observation of geomagnetic storm without Forbush-decreases, i.e. event of the $3^{\text {rd }}$ class $(\mathrm{S}-\mathrm{F})$, indicates the eastern intersection of the disturbed solar wind flow. Most likely its source will be located at the western edge of the solar disk.

\section{Conclusion}

Thus, the fulfilled analysis shows:

1. the catalog of Forbush-Storm events is a set of data on geomagnetic storms (1193 events) and decreases in CR intensity (1551 events) during 2 cycles of solar activity;

2. the catalog shows that, together with the well-known simultaneous effects in the geomagnetic field and Forbush-decreases quite often these two consequences of a disturbed solar wind flux impacts on the Earth are observed separately;

3. the ratio of these 3 classes of events varies from year to year;

4. the behavior of the interplanetary medium parameters in each of the classes of events is different and, evidently, is a consequence of 3 possible intersections of the disturbed solar wind structures by the Earth: along the western flank, in the center or along the eastern flank;

5. thereby, the use of the Forbush-Storm classification of ground-based events can be considered as a suitable device or tool for understanding how the Earth interacts with disturbed solar wind structures.

The acknowledgements. This work was supported by the RFBR grant no. 18-42-140002-p_a.

\section{References}

[1] W.D. Gonzalez, B.T. Tsurutani, A.L. Gonzalez, Space Sci. Rev., 88, 529-562 (1999).

[2] H.V. Cane, I.G. Richardson, O.C. St. Cyr, Geophys. Res. Lett., 27, 3591-3594 (2000).

[3] Yu.I. Yermolaev, M.Yu. Yermolaev, Cosmic research, 41, 105-109 (2003).

[4] J. Zhang et al. J. Geophys. Res., 112 (2007).

[5] C-C. Wu, R.P. Lepping, Adv. Space Res., 41, 335-338 (2008).

[6] D. Xu, T. Chen, X.X. Zhang, Z. Liu, Planet. and Space Sci., 57, 1500-1513 (2009). 
[7] E. Echer, W.D. Gonzalez, B.T. Tsurutani, J. Atmos. Sol.-Terr. Phys., 73, 1454-1459 (2011).

[8] N.S. Nikolaeva, Yu.I. Yermolaev, I.G. Lodkina, Geomagnetism and Aeronomy, 51, 5167 (2011).

[9] A. Goswami, Advances in Space Research, 62, 692-706 (2018).

[10] I.V. Despirak, A.A. Lyubchich, N.G. Kleimenova, Geomagnetism and Aeronomy, 59, 1-6 (2019).

[11] A.V. Belov, Proc. IAU Symposium, 257, 119-130 (2009).

[12] H.V. Cane, I.G. Richardson, I.J. Rosenwinge, J. Geophys. Res., 99, 21429-21441 (1994).

[13] M.A. Abunina et al. Geomagnetism and Aeronomy, 53, 10-18 (2013).

[14] J.J. Blanco et al. Solar Phys., 284, 167-178 (2013).

[15] P. Gololobov et al. Solar-Terrestrial Physics, 3, 20-24 (2017).

[16] CATALOGUE OF SPACE STORMS http://dbserv.sinp.msu.ru/apev/

[17] NOAA Space Weather Scales: http://www.swpc.noaa.gov/noaa-scales-explanation

[18] http://www.wdcb.ru/stp/

[19] http://www.sidc.be/silso/datafiles

[20] http://spaceweather.izmiran.ru/rus/dbs.html,

[21] L.P. Shadrina, V.P. Mamrukova, I.Ya. Plotnikov, Geomagnetism and Aeronomy, 36, 169-172 (1996).

[22] L.P. Shadrina, S.A. Starodubtsev, I.Ya. Plotnikov, Int. Geomagnetism and Aeronomy, 2(3), 45-50 (2001).

[23] K. Kudela, T. Brenkus, J. Atmosph. Solar-Terr. Phys., 66, 1121-1126 (2004).

[24] I. Parnahaj, K. Kudela, M. Kancirova, Proc. WDS'13 Contributed Papers, Part II, 1319 (2013).

[25] M. Papailiou et al. Sol. Phys., 283, 557-559 (2013).

[26] B. Badruddin et al. Space Weather, 17, 487-496 (2019).

[27] L.P. Shadrina, I.Ya. Plotnikov, S.A. Starodubtsev Forbush decreases in the absence of the magnetic storms, in Proceedings of the $9^{\text {th }}$ International Conference "Problems of Geocosmos", 08-12 Octoder 2012, St. Petersburg, Petrodvorets, Russia (2012).

[28] http://wdc.kugi.kyoto-u.ac.jp/dst realtime/index.html.

[29] T. Kuwabara et al. J. Geophys. Res., 114, A05109 (2009).

[30] F. Siteng et al. Sol. Phys., 293, 1-10 (2018). 